\title{
THE LANDAU PROBLEM FOR UNIVALENT BOUNDED NONVANISHING FUNCTIONS
}

\author{
WENFA YUAN, YONG XU AND DONGLI CHEN
}

Abstract. This paper is to inverstigate the problem of finding sup $\left|a_{0}+a_{1}+\cdots+a_{n}\right|$ for univalent holomorphic nonvanishing functions $f(z)=a_{0}+a_{1} z+\cdots$ in the unit disk $|z|<1$.

\section{Introduction}

Consider following families of the functions:

$$
\begin{aligned}
B & =\left\{f \in H(D): f(z)=a_{0}+a_{1} z+\cdots,|f(z)|<1, z \in D\right\}, \\
B_{0} & =\{f \in B: f(z) \neq 0, z \in D\}, \\
B^{s} & =\left\{f \in B_{0}: f(z) \neq 0, z \in D \text { and } f(z) \text { is univalent function }\right\}, \\
\Omega & =\left\{w \in B: w(z)=c_{1} z+c_{2} z^{2}+\cdots, z \in D\right\},
\end{aligned}
$$

where $H(D)$ denotes the set of holomorphic functions in the unit disk $D=\{z \in C:|z|<$ $1\}$. With no loss of generality we may assume that for $f \in B_{0}$ we have the normalization $a_{0}=e^{-t}, t>0$.

The function

$$
F(t, z)=\exp \left(-t \frac{1+z}{1-z}\right)=e^{-t}+\sum_{n=1}^{\infty} A_{n}(t) z^{n}
$$

plays an important role in the problem of determining $\max _{f \in B_{0}}\left|a_{n}\right|$. Landau proved

$$
\sup _{f \in B}\left|a_{0}+a_{1}+\cdots+a_{n}\right|=1+\sum_{v=1}^{n}\left(\frac{1 \cdot 3 \cdots(2 v-1)}{2 \cdot 4 \cdots(2 v)}\right)^{2}:=G_{n}, \quad n \in N .
$$

Received June 10, 2002; revised March 28, 2003.

2000 Mathematics Subject Classification. 30C50.

Key words and phrases. Univalent bounded nonvanishing functions, Landau problem, Krzyż conjecture.

Supported by the Special Science Foundation of the Educational Committee of Shaanxi Province (No:03JK066), (No:03JK065).

Supported by the Science Foundation of Xi'an University of Architecture \& Technology (No: 02BR04). 
If $f$ is univalent (say $f \in B^{s}$ ), then for every $n \in N$, we have

$$
\sup _{f \in B^{s}}\left|a_{0}+a_{1}+\cdots+a_{n}\right|<k \approx 1.616 \cdots
$$

However the result is not sharp and Lewandowski and Szynal ${ }^{[4]}$ (1999) proved the following lemma to improve the result,

Lemma. If $f \in B_{0}$, then

$$
\begin{gathered}
\left|a_{0}+a_{1}\right| \leq 2 e^{-\frac{1}{2}} \approx 1.21 \cdots \\
\left|a_{0}+a_{1}+a_{2}\right| \leq e^{-t_{0}}\left[1+2 t_{0}+\frac{t_{0}}{2\left(2-t_{0}\right)}\right] \approx 1.33 \cdots,
\end{gathered}
$$

and $t_{0}=0.66 \cdots$ is the root of equation: $-4 t^{3}+19 t^{2}-26 t+10=0$.

Based on above lemma, we give further improvement in Section 2.

\section{Main Results}

We claim the following theorem for the Landau problem of $B^{s}$.

Theroem. If $f(z) \in B^{s}$, then

$$
\begin{gathered}
\left|a_{0}+a_{1}\right| \leq 1.202 \cdots \\
\left|a_{0}+a_{1}+a_{2}\right| \leq e^{-t_{0}}\left(1+2 t_{0}^{2}\right) \leq 1.238 \cdots,
\end{gathered}
$$

with $t_{0}=1.707 \cdots$ is the root of equation: $2 t^{2}-4 t+1=0$.

Proof. By the representation formula for function $f \in B^{s}$

$$
f(z)=\exp \left(-t \frac{1+w(z)}{1-w(z)}\right)=a_{0}+a_{1} z+a_{2} z^{2}+\cdots, \quad w \in \Omega,
$$

we can get the relations:

$$
a_{0}=e^{-t}, \quad a_{1}=-2 t c_{1} e^{-t}, \quad a_{2}=-2 t e^{-t}\left[c_{2}+(1-t) c_{1}^{2}\right]
$$

Applying the inequality $\left|a_{0}\right| \leq 1,\left|a_{1}\right| \leq \frac{4\left|a_{0}\right|\left(1-\left|a_{0}\right|\right)}{1+\left|a_{0}\right|}$ and composition of univalent and let $x=\left|a_{0}\right|$, we have

$$
\left|a_{0}+a_{1}\right| \leq\left|a_{0}\right|+\frac{4\left|a_{0}\right|\left(1-\left|a_{0}\right|\right)}{1+\left|a_{0}\right|}=x+\frac{4 x(1-x)}{1+x}:=g_{1}(x)
$$

After elementary calculations of one extreme we arrive at following inequalities:

$$
\left|a_{0}+a_{1}\right| \leq g_{1}\left(x_{0}\right)=11-4 \sqrt{6} \approx 1.202 \cdots,
$$


where $x_{0}=\sqrt{\frac{8}{3}}-1$. It is easy to see that upper bound in (1) is sharp.

Using the representation (3) we have

$$
a_{0}+a_{1}+a_{2}=e^{-t}-2 t e^{-t}\left[c_{1}+c_{2}+(1-t) c_{1}^{2}\right]=e^{-t}-2 t e^{-t} \Phi\left(c_{1}, c_{2}, t\right) .
$$

Applying the inequality and composition of univalent: $\left|c_{1}\right| \leq 1,\left|c_{2}\right| \leq 2\left|c_{1}\right|\left(1-\left|c_{1}\right|\right)$, we have

$$
\begin{aligned}
& -2\left|c_{1}\right|\left(1-\left|c_{1}\right|\right) \leq c_{2} \leq 2\left|c_{1}\right|\left(1-\left|c_{1}\right|\right), \\
& \max \Phi\left(c_{1}, c_{2}, t\right)=\left|c_{1}\right|+2\left|c_{1}\right|\left(1-\left|c_{1}\right|\right)+(1-t) c_{1}^{2}=H_{1}\left(c_{1}, t\right), \\
& \min \Phi\left(c_{1}, c_{2}, t\right)=-\left|c_{1}\right|-2\left|c_{1}\right|\left(1-\left|c_{1}\right|\right)+(1-t) c_{1}^{2}=H_{2}\left(c_{1}, t\right) .
\end{aligned}
$$

There are two cases of the problem.

a). If $t \leq \frac{3}{2}, x=\left|c_{1}\right|$, then

$$
\begin{aligned}
& H_{2}\left(c_{1}, t\right)=H_{2}(x, t)=(3-t) x^{2}-3 x \\
& H_{1}\left(c_{1}, t\right)=H_{1}(x, t)=-(1+t) x^{2}+3 x .
\end{aligned}
$$

After elementary calculations of one extreme we can obtain in the following expressions

$$
\begin{gathered}
\min \Phi\left(c_{1}, c_{2}, t\right)=H_{2}\left(\frac{3}{2(3-t)}, t\right)=-\frac{9}{4(3-t)} ; \\
\max \Phi\left(c_{1}, c_{2}, t\right)=H_{1}\left(\frac{3}{2(1+t)}, t\right)=\frac{9}{4(1+t)} ; \\
e^{-t}\left(1-\frac{9 t}{2(1+t)}\right) \leq a_{0}+a_{1}+a_{2} \leq e^{-t}\left(1+\frac{9 t}{2(3-t)}\right) ; \\
-0.461 \cdots \leq a_{0}+a_{1}+a_{2} \leq 1.227 \cdots
\end{gathered}
$$

Hence

$$
\left|a_{0}+a_{1}+a_{2}\right| \leq e^{-t}\left(1+\frac{9 t_{0}}{2\left(3-t_{0}\right)}\right) \approx 1.227 \cdots
$$

b). From the inequality $\left|c_{2}\right| \leq 2\left|c_{1}\right|\left(1-\left|c_{1}\right|\right)$ and if $t>\frac{3}{2}, x=\left|c_{1}\right|$, then we have

$$
\left|a_{0}+a_{1}+a_{2}\right| \leq e^{-t}\left\{2 t(t-3) x^{2}+6 t x+1\right\}:=e^{-t} g_{2}(x) .
$$

We arrive at following after elementary calculations of one extreme

$$
g_{2}(x) \leq g_{2}(1)=e^{-t}\left(1+2 t^{2}\right) \leq 1.238 \cdots
$$

According to $\mathbf{a}$ ) and $\mathbf{b}$ ) we obtain

$$
\left|a_{0}+a_{1}+a_{2}\right| \leq e^{-t} \begin{cases}1+\frac{9 t}{2(3-t)}, & t \leq \frac{3}{2} \\ 1+2 t^{2}, & t>\frac{3}{2}\end{cases}
$$


Hence

$$
\left|a_{0}+a_{1}+a_{2}\right| \leq e^{-t_{0}}\left(1+2 t_{0}^{2}\right) \leq 1.238 \cdots, \quad \text { where } t_{0}=1.707 \cdots
$$

Remark. We conjecture that for any $f \in B^{s}$ and $n \in N$ there exists an absolute constant $L>1$ such that

$$
\sup _{f \in B^{s}}\left|a_{0}+a_{1}+\cdots+a_{n}\right| \leq L<k \cong 1.616 \cdots
$$

\section{References}

[1] J. X. Chen, One inequality in theory of subordination, Chinese Sci. Bull. 4(1982), 193-197.

[2] J. A. Hummel, S. Scheinberg and L. Zalcman, A coefficient problem for bounded nonvanishing functions, J. Math. Anal. Appl. 31(1977), 169-190.

[3] Z. Lewandowski and J. Szynal, On the Krzyż conjecture and related problem, in: Laine, Martio (Eds), XVIth Rolf Nevalinna Colloquim, Walter de Gruyter, Berlin, 1996, 257-268.

[4] Z. Lewandowski and J. Szynal, The Landau problem for bounded nonvanishing functions, J. Comput. Appl. Math. 105(1999), 367-369.

[5] D. L. Tan, Estimate coefficient for bounded nonvanishing functions, Chinese Ann. of Math. 4(1983), 97-104.

[6] Z. Z. Zou, One the Landau problem for bounded nonvanishing functions, Ann. Univ. Mariae Curie-Sklodowska Sect. A 52(1998), 37-46.

College of Science, Xi'an University of Architecture \& Technology, Xi'an 710055, P. R. China.

Department of Applied Mathematics, Northwestern Polytechnical University, Xi'an 710072, P. R. China.

College of Science, Xi'an University of Architecture \& Technology, Xi'an 710055, P. R. China. 